\title{
Multiple Description Coding for Uncompressed Video Streaming over 60GHz Networks
}

\author{
Zhifeng $\mathrm{He}$ \\ Department of Electrical and Computer \\ Engineering, Auburn University \\ 200 Broun Hall, Auburn, AL 36849-5201 USA \\ zzh0008@tigermail.auburn.edu
}

\author{
Shiwen Mao \\ Department of Electrical and Computer \\ Engineering, Auburn University \\ 200 Broun Hall, Auburn, AL 36849-5201 USA \\ smao@ieee.org
}

\begin{abstract}
Cognitive radio (CR) has been recognized as an important technology for enhancing spectrum efficiency. While many dynamic spectrum access techniques are developed to better utilize the allocated spectrum, the massive unlicensed bandwidth in the $60 \mathrm{GHz}$ band also provides great potential for supporting new bandwidth intensive applications. In this paper, we investigate the problem of streaming uncompressed High Definition (HD) videos over $60 \mathrm{GHz}$ networks. We present a multiple description (MD) coding (MDC) technique based on Priority Encoding Transmission (PET) that exploits the different significance of the pixel bits, and an interleaving based transmission scheme to combat the bursty losses due to blockage. A nonlinear integer programming problem is formulated and solved for determining the optimal parameters. The proposed scheme is cognitive and adaptive to the dynamic $60 \mathrm{GHz}$ link conditions for enhanced video quality. The performance of the proposed scheme is validated with simulations.
\end{abstract}

\section{Categories and Subject Descriptors}

C.2.1 [Computer-Communication Networks]: Network Architecture and Design-Wireless communication; Network communications;

C.2.2 [Computer-Communication Networks]: Network Protocols-Applications

\section{Keywords}

60GHz networks; multiple description coding; optimization; quality of service; video streaming

\section{INTRODUCTION}

With the dramatic advances in wireless networking technology, there is an exponentially increasing demand for wireless data service. In particular, mobile video is predicted to grow at a compound annual growth rate (CAGR) of $90 \%$

Permission to make digital or hard copies of all or part of this work for personal or classroom use is granted without fee provided that copies are not made or distributed for profit or commercial advantage and that copies bear this notice and the full citation on the first page. Copyrights for components of this work owned by others than ACM must be honored. Abstracting with credit is permitted. To copy otherwise, or republish, to post on servers or to redistribute to lists, requires prior specific permission and/or a fee. Request permissions from permissions@acm.org. ACM CRAB '13 Miami, FL USA

Copyright 2013 ACM 978-1-4503-2368-0/13/10 ...\$15.00.

http://dx.doi.org/10.1145/2508478.2508485. from 2011 to 2016. Cognitive radio (CR) has been recognized as an important technology for enhancing spectrum efficiency, while many dynamic spectrum access techniques are developed to better utilize the allocated spectrum [17]. On the other hand, millimeter wave (mm-wave) communications in the $60 \mathrm{GHz}$ band has gained considerable interest from academia, industry, and standards bodies [10]. This is due to the huge unlicensed bandwidth (i.e., up to $7 \mathrm{GHz}$ ) that is available in most parts of the world. This massive unlicensed bandwidth provides great potential to meet the surging wireless video demand, as well as supporting new bandwidth demanding applications [3,14].

Recently, the problem of transmitting uncompressed High Definition (HD) videos via $60 \mathrm{GHz}$ channels has attracted intensive interest. The immense bandwidth of the $60 \mathrm{GHz}$ channel enables streaming of uncompressed HD videos with high data rates [4], such as the 1080p video, which has $1920 \times 1080$ pixels (each of which has 24 bits) per video frame and the frame size is about $6 \mathrm{MBs}$. Such high speed wireless links can not only replace the cumbersome HDMI cables, but also relieve the computational burden for video decoding at the display devices (e.g., projectors or HDTVs).

Although the larger bandwidth is a great advantage of $60 \mathrm{GHz}$ networks, the $60 \mathrm{GHz}$ transmissions are highly directional to overcome the high attenuation, making it susceptible to blockage of the line-of-sight path by obstacles or pedestrians. Thus the memory of the channel poses a negative effect on the performance of the system, and channel conditions such as packet loss rate may change over time [16]. How to adjust the operation parameters of the transmission system is critical to maintain a certain level of QoS under such conditions.

In this paper, we investigate the problem of streaming uncompressed HD video over $60 \mathrm{GHz}$ networks. Considering the fact that bits of different positions in a pixel has different level of significance on recovering the original video frame [5], it is desirable to improve the quality of reconstructed video frames by offering more protection for the more important bits. We adopt multiple description (MD) coding (MDC) to combat the lossy $60 \mathrm{GHz}$ channel. In $\mathrm{MDC}$, a video is encoded into multiple descriptions, each can guarantee a low but acceptable video quality. More important, the more descriptions received, the better the video quality. MDC has been used mostly for compressed videos in lossy wireless networks in the literature $[7,8]$.

In this paper, we adopt MD coding for uncompressed videos, exploiting the different significance of the different bits in a pixel. In particular, in order to ensure the quality 
Table 1: Notation

\begin{tabular}{|c|c|}
\hline Symbol & Description \\
\hline$M$ & Number of rows of the image matrix \\
\hline$N$ & Number of columns of the image matrix \\
\hline$C$ & Number of rows of the sub-image matrix \\
\hline$D$ & $\begin{array}{l}\text { Number of columns of the sub-image } \\
\text { matrix }\end{array}$ \\
\hline$Q$ & Number of sub-images of an image \\
\hline$S$ & $\begin{array}{l}\text { Number of segments the } 8 \text { bits are } \\
\text { divided into }\end{array}$ \\
\hline$m_{i}$ & Length of the $i$-th segment, $1 \leq i \leq S$ \\
\hline$k$ & Number of bits constituting a symbol \\
\hline$g_{i}$ & $\begin{array}{l}\text { Number of groups the data symbols of } \\
\text { stream } i \text { are divided }\end{array}$ \\
\hline$L$ & Code word length of the RS code \\
\hline$P_{e}(G)$ & $\begin{array}{l}\text { Probability of an error occurring in the } \\
\text { good state }\end{array}$ \\
\hline$P_{e}(B)$ & $\begin{array}{l}\text { Probability of an error occurring in the } \\
\text { bad state }\end{array}$ \\
\hline$P_{g \mid b}$ & $\begin{array}{l}\text { Channel state transition probability from } \\
\text { good to bad }\end{array}$ \\
\hline$P_{b \mid g}$ & $\begin{array}{l}\text { Channel state transition probability from } \\
\text { bad to good }\end{array}$ \\
\hline$H$ & Number of images interleaved together \\
\hline$\Delta$ & $\begin{array}{l}\text { Maximum tolerable time of recovering } \\
\text { the image }\end{array}$ \\
\hline$\Lambda_{l}$ & Minimum packet size \\
\hline$\Lambda_{l}$ & Maximum packet size \\
\hline
\end{tabular}

of the reconstructed video, we divide the bits in a pixel into multiple segments according to their respective significance in enhancing video quality, and provide higher protection to bits of greater importance by assigning more forward error correction (FEC) symbols to them. This way, the decoder has a higher probability of recovering the most important bits, which affect the quality of the reconstructed image in a largest extent. Such an MD coder is termed MD-FEC. How to partition the bits, i.e., how many segments the bits should be divided into, and how many bits a particular segment should have, affects the significance of a particular segment and the probability of decoding the segment.

To combat the bursty errors while transmitting over the $60 \mathrm{GHz}$ channel, interleaving among multiple video frames is applied in our scheme. We formulate a nonlinear integer programming problem, which can be solved to find the optimal partition of the pixel bits in the MD coder, as well as the optimal interleaving depth, for given channel conditions. The proposed scheme is cognitive and adaptive to the dynamic $60 \mathrm{GHz}$ link conditions for enhanced video quality. It is evaluated with simulations and is shown to outperform an existing scheme with considerable gains.

The remainder of the paper is organized as follows. Related work is reviewed in Section 2. We present the proposed MD coding and transmission scheme in Section 3 and simulation results in Section 4. Section 5 concludes the paper. The notation used in this paper is summarized in Table 1.

\section{RELATED WORK}

In this section, we briefly review related work on video transmission over $60 \mathrm{GHz}$ channels, and two MD-FEC tech- niques and Reed-Solomon coding, which are the basis of our proposed scheme.

Considering the similarity in the most important bits of the neighboring pixels in an image, the authors of [5] propose a technique to correct the error in the most important bits in a pixel by comparing those bits of the pixel with the bits at the same positions in its neighboring pixels. Shao et al. in [12] also develop a method to recover the bits of a pixel using the neighboring pixels. With this scheme, the neighboring pixels are encapsulated into different packets that are transmitted separately, so that even if a pixel is lost it can still be approximated by other received packets containing the neighboring pixels. However, there is no measures on providing better protection for the most important bits during transmission, which affects the quality of reconstructed video frames.

In [13], an Uncompressed Video streaming over Wireless (UVoW) system is proposed for transmission of uncompressed video on $60 \mathrm{GHz}$ channels. In this system, the 8 bits representing a pixel are divided into two 4-bit parts according to their significance in recovering the video: MSB (most significant bits) and LSB (least significant bits). The MSB portions are better protected then the LSB portions by being transmitted through channel of better conditions. The encoder retransmits the MSB part if it has error, but corrupted LSB parts will not be retransmitted. However, there is no evidence that the system achieves the best performance by cutting the 8 bits into two 4 -bit segments, and there is no investigation on, when channel condition changes, how to adjust the operational parameters to make it adaptive to the changing channel conditions.

In [2], Bosco develops a cross-layer adaptive scheme for $\mathrm{HD}$ videos streaming over $60 \mathrm{GHz}$ channels, where the sender adjusts the modulation and coding schemes (MCS) in response to channel variations to maintain good video quality. A method of cooperation with relay for transmission of HD videos over $60 \mathrm{GHz}$ channels is developed in [4]. The source and relay use different error correcting codes so that error correcting performance can be enhanced, and spatial diversity gain is obtained by using the relay. As in [13], the message is also cut into two fixed parts and the optimal segmentation of the message is out of concern in this paper.

MD video coding has been shown to be highly effective for video over lossy networks $[6,8,9,15]$. Among the many MD coding techniques, MD-FEC is a popular one based on Priority Encoding Transmission (PET) [1], which assigns different levels of protection to data segments according to their respective priority defined by the user [15]. The higher the priority, the smaller number of packets the decoder needs to decode it, so that the higher priority segments have a greater probability of being decoded. The basis of this algorithm is Reed-Solomon (RS) coding, which is a sub-class of the Bose-Chadhuri-Hocquenghem (BCH) codes. RS coding provides an effective way of recovering lost data symbols from a portion of data symbols received [11].

\section{SYSTEM MODEL, PROBLEM STATEM- ENT AND SOLUTION PROCEDURE}

We consider streaming uncompressed HD video over a $60 \mathrm{GHz}$ wireless link (e.g., from a storage device to an HDTV or a video projector). The MD-FEC coding scheme for uncompressed HD video is introduced in Section 3.1. The 


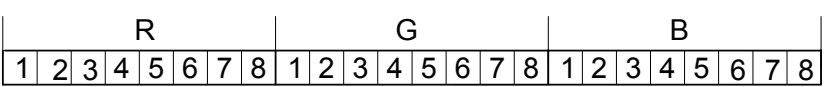

Figure 1: Structure of a pixel in the uncompressed video.

$60 \mathrm{GHz}$ channel model and the interleaving based transmission strategy is presented in Section 3.2. Then we present our problem formulation in Section 3.3 and solution procedure in Section 3.4.

\subsection{MD-FEC Coding of Uncompressed Video}

Consider an uncompressed video frame of $M \times N$ pixels. Each pixel consists of $3 \times 8=24$ bits, while each 8 -bit block corresponding to one of the $\mathrm{R} / \mathrm{G} / \mathrm{B}$ components as shown in Fig. 1. The $j$-th bit in each of the 8-bit block represents a value of $2^{8-j}$. Obviously, the $j$-th bit is more important than the $(j+i)$-th bit, $i \geq 1$, because it has a greater influence on the color depth of the R/G/B component of the pixel.

Next we divide the frame evenly into $Q$ sub-images, each of which has $C \times D$ pixels, i.e., $Q=\frac{M N}{C D}$, for the purpose of:

- the packet size will not become too large so that the packet transmission time can be fitted in the unit time slot in our channel model;

- the number of data symbols of a data stream will not become too large so that RS codes can be applied to the data symbols.

Now let's take the $r$-th sub-image as an example; each of the following procedures will be performed on the other subimages as well. Each of the 8 bits representing the $\mathrm{R} / \mathrm{G} / \mathrm{B}$ color in a pixel is divided into $S$ segments, $1 \leq S \leq 8$, and the length (i.e., the number of bits) of segment $i$, is denoted as $m_{i}, 1 \leq i \leq S$. Rearrange all the $i$-th segments of the $\mathrm{R} / \mathrm{G} / \mathrm{B}$ color of all the pixels in a sub-image into a new segment called data stream $i$, which has $\frac{3 m_{i} M N}{Q}$ bits. We define the significance of data stream $i$ of sub-image $r$ as the summation of the values of the data bits in segment $i$ of sub-image $r$, and denote it as $w_{i}^{r}$.

$$
w_{i}^{r}=\frac{3 M N}{Q} \sum_{l=1}^{m_{i}-1} 2^{8-\sum_{j=1}^{i-1} m_{j}-l} .
$$

Due to uncompressed video, each pixel in the frame is equally important. We have $w_{i}^{r}=w_{i}^{t}$ for any $r \neq t, 1 \leq$ $r \leq Q, 1 \leq t \leq Q$. Fig. 2 shows an example of cutting each of the 8 bits of a pixel in a sub-image into 3 segments and combining all the first segments into data stream 1 of the sub-image.

From (1), we can see that $w_{i}^{r} \geq w_{i+1}^{r}$, for all $1 \leq r \leq Q$, $1 \leq i \leq S-1$, which implies that recovering data stream $i$ is more important than recovering data stream $(i+1)$ for the purpose of enhancing the quality of reconstructed frames at the receiver. Therefore, we assign higher protection to data stream $i$ than data stream $(i+1)$ to make the probability of recovering data stream $i$ higher than that of recovering data stream $(i+1)$. We illustrate how to achieve this goal below.

Firstly we let $k$ bits in a data steam form a data symbol. For example, we can take $k=8$ for the convenience of computer processing. Then data stream $i$ can be represented by $\frac{3 m_{i} M N}{Q k}$ data symbols. These $\frac{3 m_{i} M N}{Q k}$ symbols of data stream $i$ is further divided into $g_{i}$ groups, each of which has

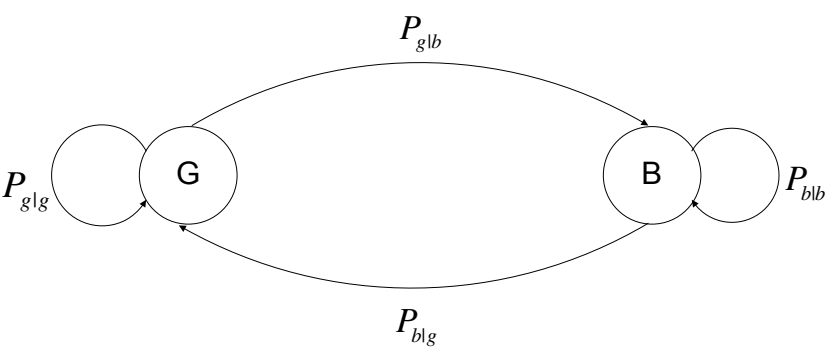

Figure 4: The Gilbert-Elliot link model.

$\frac{3 m_{i} M N}{Q k g_{i}}$ data symbols. We apply RS code to each group so that the total number of symbols (including both data and FEC symbols) is $L, L \leq 2^{k}-1$, which is a constraint of RS coding.

This way, the number of FEC symbols assigned to each group of data stream $i$ is $L-\frac{3 m_{i} M N}{Q k g_{i}}$. The $j$-th symbol of all the groups of all the data streams compose the $j$-th description of the frame, and is transmitted in one packet. This procedure is illustrated in Fig. 3. To assign higher protection to data stream $i$ than data stream $(i+1)$, the error correcting capacity, or the amount of FEC symbols for data stream $i$ should be greater than that of data stream $(i+1)$, i.e.,

$$
L-\frac{3 m_{i} M N}{Q k g_{i}} \geq L-\frac{3 m_{i+1} M N}{Q k g_{i+1}},
$$

which implies that $\frac{m_{i}}{g_{i}} \leq \frac{m_{i+1}}{g_{i+1}}$.

As shown in Fig. 3, each description (or, packet) consists of video data bits and FEC bits from all the data streams, and is equally important for reconstructing the frame. The quality of the reconstructed frame is proportional to the number of descriptions received. If $\frac{3 m_{1} M N}{Q k g_{1}}$ or more errorfree descriptions are received, then all the groups of data stream 1 can be decoded, which guarantees a basic quality for this video frame. In general, if the decoder receives at least $\frac{3 m_{i} M N}{Q k g_{i}}$ error-free descriptions, then all the data streams up to $i$ can be decoded, resulting in an improved quality for the frame. When more than $\frac{3 m_{S} M N}{Q k g_{S}} \leq L$ errorfree descriptions are received, the video frame can be reconstructed without any error.

\subsection{GHz Channel and Transmission Sched- ule}

After MD-FEC coding, the video packets are then transmitted over the $60 \mathrm{GHz}$ link from the sender (e.g., a storage device) to the receiver (e.g., an HDTV or video projector). As discussed, the $60 \mathrm{GHz}$ channel is highly directional and susceptible to blockage of the link-of-sight path due to obstacles. Therefore, we model the $60 \mathrm{GHz}$ link with the Gilbert-Elliot (GE) model, which is a discrete-time two-state Markov chain as shown in Fig. 4. The two states are: good state (denoted as $G$ in the figure) and bad state (denoted as $B)$. The probabilities that an error occurring at the good state and bad state are denoted as $P_{e}(G)$ and $P_{e}(B)$, respectively. The transition probabilities from $G$ to $B$ and from $B$ to $G$ are $P_{g \mid b}$ and $P_{b \mid g}$, respectively. The transmission time of a packet over the $60 \mathrm{GHz}$ link is less than or equal to the duration of one time slot.

When multiple beam paths are available between the transmitter and receiver (e.g., a direct path and a second path 


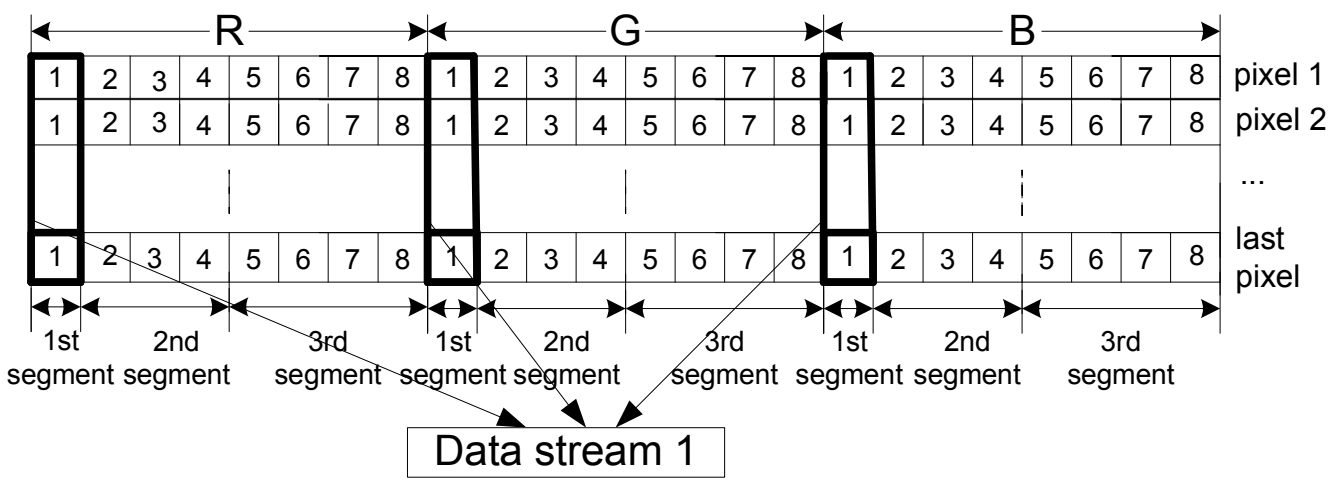

Figure 2: Structure of a data stream, assuming $S=3$.

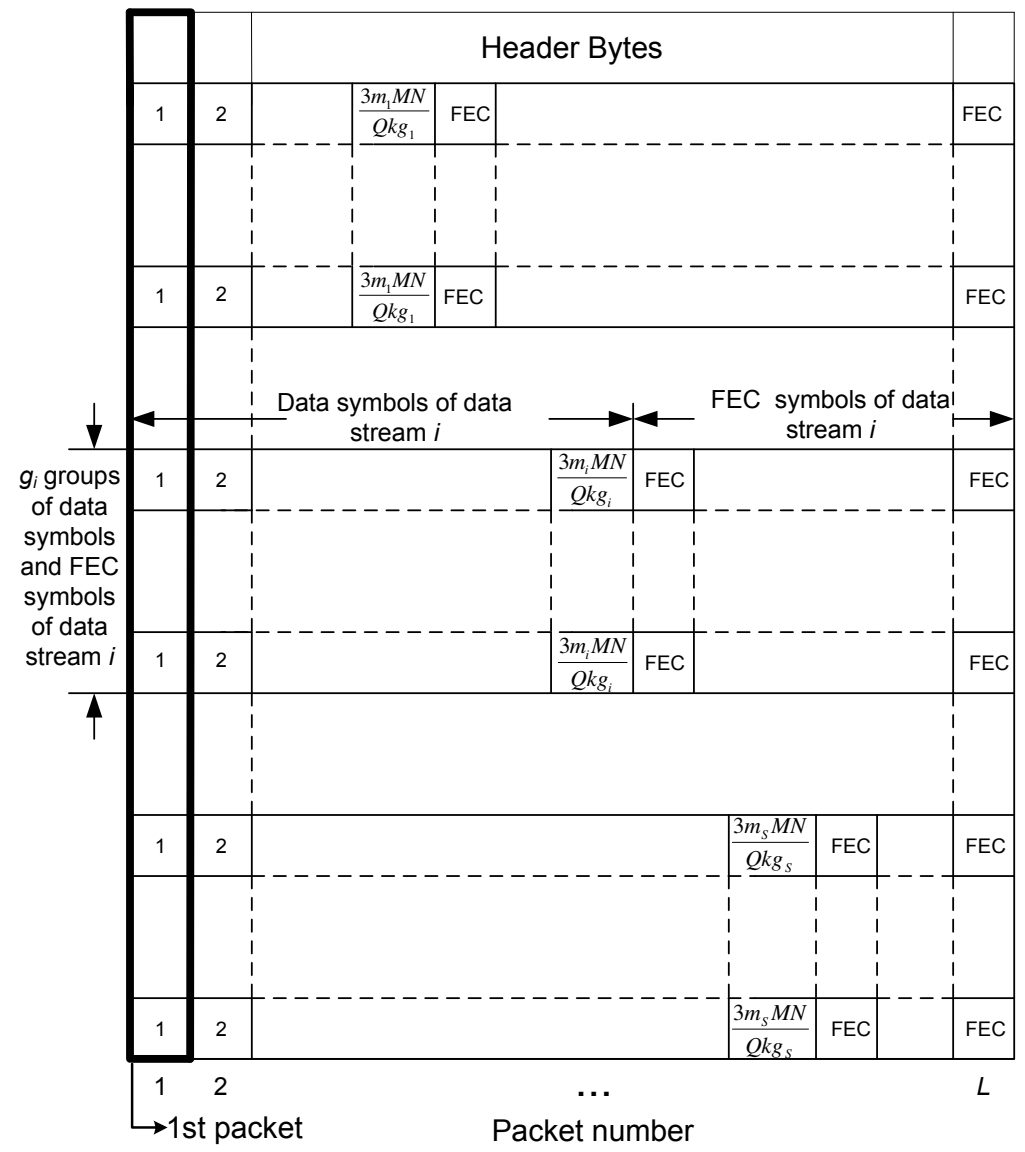

Figure 3: Structure of the packets of a sub-image.

due to reflection on the wall), the multipath transport technique can be used for the MD video packets [6]. In this paper, we focus on the single beam path case, while the proposed technique can be extended to the case of multiple beam paths. Recall that the MD-FEC coding can ensure a minimum video quality if at least $\frac{3 m_{1} M N}{Q k g_{1}}$ error-free packets are received for a frame. However, if most of the packets are transmitted within a bad period, it is possible that less than $\frac{3 m_{1} M N}{Q k g_{1}}$ packets can be received and the video frame cannot be decoded. In order to reduce the bursty error rate, we adopt interleaving to the packets of multiple consecutive frames.
The interleaving scheme is illustrated in Fig. 5. The interleaving depth is the time interval between the transmissions of two consecutive packets of a sub-image. As shown in the figure, the interleaving depth is set to $Q H$, while $H$ is a nonnegative integer. We can adjust $H$ to find the best interleaving depth for different channel conditions in terms of $P_{e}(G)$, $P_{e}(B)$, and the transition probabilities. when the average bad period $T_{o f f}$ is large, we can increase $Q H$ by increasing $H$. The probability that multiple consecutive packets of a sub-image are transmitted within the bad period can be reduced. Since RS coding is applied in our scheme, the probability of failing to decode data stream $i$ of sub-image $X$ 


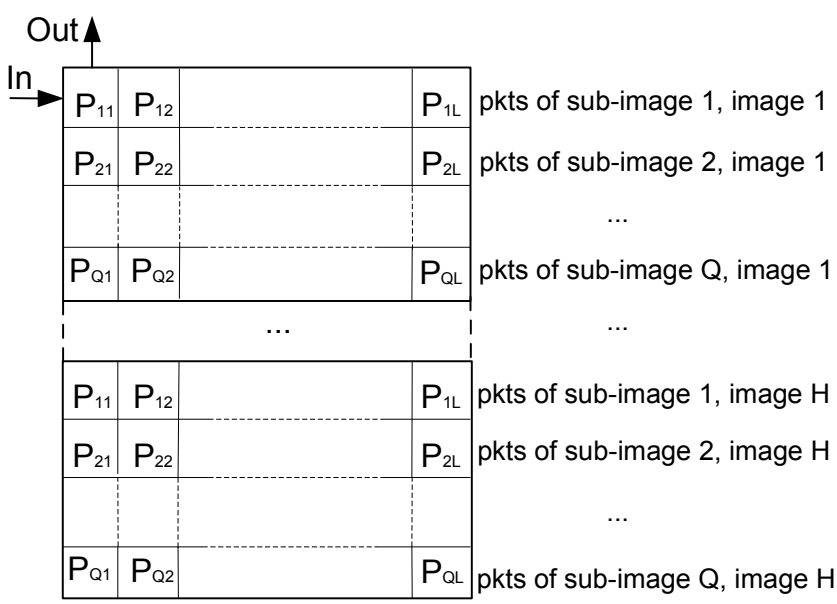

Figure 5: Structure of the interleaver.

of image $Y$ is the probability that the decoder receives less than $\frac{3 m_{i} M N}{Q k g_{i}}$ error-free data packets, which can be expressed as $[16]$

$$
\begin{aligned}
P_{i}^{X Y}= & \sum_{d=0}^{Z} P_{z}(d)\left\{\sum _ { e _ { b } = 0 } ^ { d } \left[C_{d}^{e_{b}} P_{e}^{e_{b}}(B)\left(1-P_{e}(B)\right)^{d-e_{b}}\right.\right. \\
& \left.\left.\sum_{e_{g}=\max \left\{0, t+1-e_{b}\right\}}^{Z-d} C_{Z-d}^{e_{g}} P_{e}^{e_{g}}(G)\left(1-P_{e}(G)\right)^{Z-d-e_{g}}\right]\right\},
\end{aligned}
$$

where $P_{Z}(d)$ is the probability that given the GE channel is observed at $Z$ continuous time slots, the probability that the channel is in the bad state for $d$ time slots; $Z$ is the block code length of the error correcting code being used and thus $Z=L=2^{k}-1$ in our example; $e_{b}$ and $e_{g}$ denote the numbers of packets in error when the channel is the bad state and good state, respectively; $t$ is the error correcting capability of the error correcting code for data stream $i$ and thus $t=L-\frac{3 m_{i} M N}{Q k g_{i}}$ in our case; and $P_{i}^{X Y}=P_{i}^{X^{\prime} Y^{\prime}}=P_{i}$, for any $X \neq X^{\prime}$ and $Y \neq Y^{\prime}$, due to uncompressed videos.

For brevity, retransmission is not explicitly implemented in our system. Therefore, the transmission time for all the packets of a data stream $i$ is $L H Q$ time slots. A retransmission scheme can be translated to reduced $P_{e}(G)$ and $P_{e}(B)$, but the transmission times for the data streams could be longer [13].

\subsection{Problem Statement}

Now we are ready to formulate the problem of MD video over $60 \mathrm{GHz}$ networks. The goal is to minimize the expected Mean Square Error (i.e., MSE or $\sqrt{\mathrm{MSE}}$ ) of the reconstructed video frames, which is a function of the various design factors as follows. First, the MSE of a reconstructed frame is defined as

$$
\mathrm{MSE}=\frac{1}{3 M N} \sum_{i=1}^{M} \sum_{j=1}^{N} \sum_{m=1}^{3}\left[I(i, j, m)-I^{\prime}(i, j, m)\right]^{2} .
$$

In (4), $I(i, j, m)$ is a pixel in the original video frame and $I^{\prime}(i, j, m)$ is the pixel at the same location in the recon- structed video frame. It follows that

$$
\begin{aligned}
& \mathbb{E}\{\sqrt{\mathrm{MSE}}\} \\
& =\mathbb{E}\left\{\sqrt{\frac{1}{3 M N} \sum_{q=1}^{Q}\left\{\sum_{i=1}^{C} \sum_{j=1}^{D} \sum_{m=1}^{3}\left[I(i, j, m)-I^{\prime}(i, j, m)\right]^{2}\right\}}\right\} \\
& =\mathbb{E}\left\{\sqrt{\frac{1}{3 M N} \sum_{q=1}^{Q}\left[\sum_{i=1}^{C} \sum_{j=1}^{D} \sum_{m=1}^{3}\left(\sum_{r=T_{q}}^{S_{q}} \frac{Q}{3 M N} w_{q}^{r}\right)^{2}\right]}\right\} \\
& =\mathbb{E}\left\{\sqrt{\frac{1}{3 M N} \sum_{q=1}^{Q}\left[3 C D\left(\sum_{r=T_{q}}^{S_{q}} \frac{Q}{3 M N} w_{q}^{r}\right)^{2}\right]}\right\} \\
& =\mathbb{E}\left\{\sqrt{\frac{1}{Q} \sum_{q=1}^{Q}\left(\sum_{r=T_{q}}^{S_{q}} \frac{Q}{3 M N} w_{q}^{r}\right)^{2}}\right\} \\
& =\sum_{T_{1}=1}^{S_{1}} \cdots \sum_{T_{Q}=1}^{S_{Q}}\left\{\left[\prod_{q=1}^{Q} P\left(x_{T_{q}-1} \leq X_{q}<x_{T_{q}}\right)\right] .\right. \\
& \left.\sqrt{\frac{1}{Q} \sum_{q=1}^{Q}\left(\sum_{r=T_{q}}^{S_{q}} \frac{Q}{3 M N} w_{q}^{r}\right)^{2}}\right\} \\
& =\sum_{T_{1}=1}^{S_{1}} \cdots \sum_{T_{Q}=1}^{S_{Q}}\left\{\left[\prod_{q=1}^{Q}\left(P_{T_{q}}-P_{T_{q}-1}\right)\right] .\right. \\
& \left.\sqrt{\frac{1}{Q} \sum_{q=1}^{Q}\left(\sum_{r=T_{q}}^{S_{q}} \frac{Q}{3 M N} w_{q}^{r}\right)^{2}}\right\}
\end{aligned}
$$

Where $S_{q}, 1 \leq q \leq Q$ is the total number of segments that each pixel of the sub-image $q$ is divided into; $T_{q}$ is the first segment of sub-image $q$ that cannot be decoded; $X_{q}$ is the number of packets of sub-image $q$ that are received and not corrupted by error; $x_{T_{q}}$ is the number of data symbols required to decode $T_{q}$ (and denote $x_{0}=0$ ); $P\left(X_{q}<x_{T_{q}}\right)$ is the probability that $X_{q}$ is less than $x_{T_{q}}$, such that $P\left(X_{q}<x_{T_{q}}\right)=P_{T_{q}}$, where $P_{T_{q}}$ is the probability that data $T_{q}$ cannot be decoded, as given in (3).

We then formulate the following nonlinear integer programming (NLIP) problem.

$$
\begin{aligned}
\text { minimize } & \mathbb{E}\{\sqrt{\mathrm{MSE}}\} \\
\text { subject to: } & \frac{m_{i}}{g_{i}} \leq \frac{m_{i+1}}{g_{i+1}}, \text { for all } i \\
& \frac{3 m_{S_{q}} M N}{Q k g_{S_{q}}} \leq L, \text { for all } q \\
& L \leq 2^{k}-1 \\
& \sum_{i=1}^{S_{q}} m_{i}=8, \text { for all } q \\
& L H Q \leq \Delta \\
& \Lambda_{l} \leq \sum_{i=1}^{S_{q}} \frac{g_{i} k}{8} \leq \Lambda_{u}, \text { for all } q .
\end{aligned}
$$

Constraint (11) is due to the fact that the transmission time of all the packets of an image must not be larger than a 
tolerable transmission delay, i.e., the delay bound $\Delta$. Constraint (12) is for the purpose of making the size of a packet adequate for transmission after adding header bytes to the packets, i.e., within the range of the minimum packet size $\Lambda_{l}$ and the maximum packet size $\Lambda_{u}$.

\subsection{Solution Strategy}

The formulated problem aims to minimize the expected $\sqrt{\mathrm{MSE}}$ given in (5). This goal is achieved by tuning the control parameters $S,\left\{m_{1}, m_{2}, \cdots, m_{S_{q}}\right\},\left\{g_{1}, g_{2}, \cdots, g_{S_{q}}\right\}$, for all $q$, and $H$ to find the optimal partition of the pixel bits for MD coding and the optimal interleaving depth for transmission.

Checking out the search space for the optimal solution, it can be seen that: (i) the feasible values for $S_{q}$ is between 1 and 8; (ii) due to constraint (10), the $m_{i}$ 's are all small integers; and (iii) $H$ is also limited by the maximum delay constraint (11). The parameters that dominate the search space of the problem are $\left\{g_{1}, g_{2}, \cdots, g_{S_{q}}\right\}$, for all $q$, since the packet size can be a value between hundreds to thousands of bytes. This motivates us to reduce the search space by "taking out" $\left\{g_{1}, g_{2}, \cdots, g_{S_{q}}\right\}$, for all $q$, such that the search space of the problem will be small enough to apply an exhaustive search for the remaining parameters. This way, the problem can be reduced to a sub-optimal problem having $S_{q}$, $\left\{m_{1}, m_{2}, \cdots, m_{S_{q}}\right\}$, and $H$ as its optimization parameters.

In our solution strategy, we reduce constraint (12) into $\sum_{i=1}^{S_{q}} \frac{g_{i} k}{8}=\Lambda$, where $\Lambda$ is a constant, for all $q$. For a particular $S_{q}$, for all $q$, we have $g_{i}=\frac{8 \Lambda}{k S_{q}}$, for all $i$. Furthermore, constraint (7) can be transformed to $m_{i} \leq m_{j}$, for all $i<j$. Then the problem is reduced to a sub-optimal problem of finding the optimal $\left\{m_{1}, m_{2}, \cdots, m_{S_{q}}\right\}$ and $H$ under a specific $S_{q}$ value. The search space of the sub-optimal problem is now much smaller than that of the original problem, so that exhaustive search can be applied. The ideas of finding the best bits-partition scheme and interleaving depth are still maintained in the sub-optimal problem, thus the proposed scheme still being adaptive to channel dynamics. Note that for the case where $\frac{8 \Lambda}{k S_{q}}$ is not an integer, we take $g_{i}=\left\lfloor\frac{8 \Lambda}{k S_{q}}\right\rfloor$, for all $1 \leq i \leq S_{q}$ and the remaining space in the packet left by the data symbols are padded by redundancy.

In addition, we also aim to reduce the number of loops caused by $Q$, since a large number of loops will seriously drag down the speed of the exhaustive search algorithm, as can be seen from (5). We cut the HD video image into many small images (different from the sub-images mentioned above) and take each of the small images as an intact image, and then interleaving is applied to these small images. Thus $Q=1$ in the exhaustive search algorithm and our following simulations. And we omit the subscript $q$ for brevity in the following. This way, the number of loops is greatly reduced and simulation speed is greatly improved, and the simulation result shows the correctness of the solution strategy obtained from the exhaustive search algorithm. The performance of this solution strategy will be demonstrated in the performance evaluation section.

\section{PERFORMANCE EVALUATION}

In this section, we evaluate the performance of the proposed scheme. In the simulations, we use $1920 \times 1080 \mathrm{HD}$ uncompressed images with 24 bits per pixel (i.e., 8 bits for each $\mathrm{R} / \mathrm{G} / \mathrm{B}$ component), instead of a real $\mathrm{HD}$ video stream.
Table 2: Simulation Parameters

\begin{tabular}{ll}
\hline Parameters & Value \\
\hline$M$ & 1080 \\
$N$ & 1920 \\
$C$ & 60 \\
$D$ & 80 \\
$k$ & 6 \\
$\Delta$ & $10^{6}$ time slots \\
$L$ & 51 \\
$H$ & $1 \sim 5$ \\
$\Lambda_{l}$ & 800 Bytes \\
$\Lambda_{u}$ & 2000 Bytes \\
$\sum_{i=1}^{S} \frac{g_{i} k}{8}$ & 900 Bytes \\
\hline
\end{tabular}

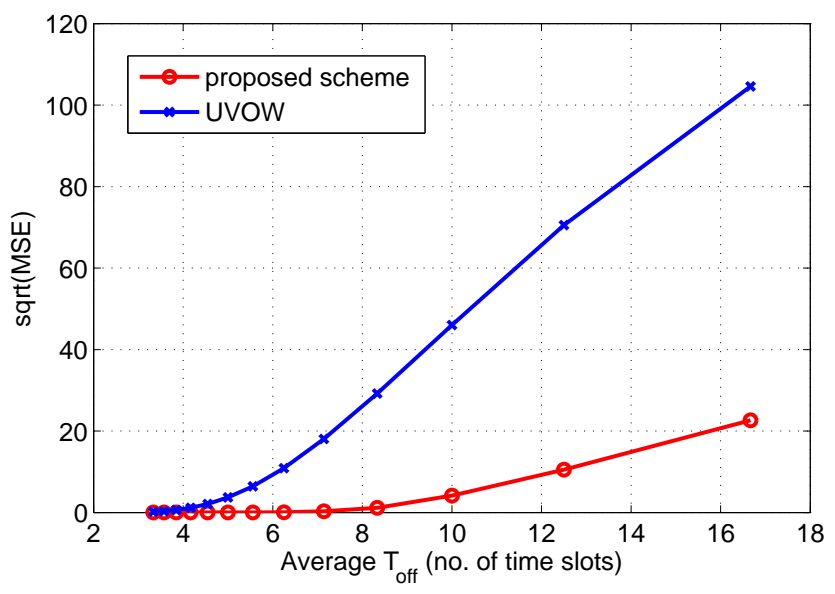

Figure 6: $\sqrt{\text { MSE }}$ vs. average bad period of the 60 GHz channel $T_{o f f}$.

The simulation parameters are shown in Table 2. We compare the proposed scheme with UVoW presented in [13], as discussed in Section 2. Note that ARQ is not employed in both schemes for a fair comparison.

We firstly compare the expected $\sqrt{\mathrm{MSE}}$ achieved by the proposed scheme and UVoW, by varying the average duration of the channel bad period. The results are plotted in Fig. 6. Here $P_{b \mid g}$ is increased from 0.06 to 0.3 with unit increment 0.02. Note that using our proposed scheme, the best combination of $\left\{S, m_{1}, m_{2}, \cdots, m_{S}, H\right\}$, which leads to the lowest expected $\sqrt{\mathrm{MSE}}$ under the given channel condition, can be found for each different average $T_{\text {off }}$ value. We can see from the figure that the expected $\sqrt{\mathrm{MSE}}$ of the proposed scheme is lower than that of UVoW for the entire range. When channel condition is good (i.e., the average $T_{\text {off }}$ is less than 4 ), the performance gap between the two schemes is not remarkable. However, as the channel condition degrades (i.e., average $T_{\text {off }}$ is greater than 4 ), the performance gap grows, for the reason that the packet loss rate grows thus it is of greater significance to provide strong protection to the most important bits. The proposed scheme can adapt to changing channel conditions, to strengthen the protection for the most important bits and increase the interleaving depth to combat the temporarily blocked channel. On average, the proposed scheme achieves 19.6055 lower $\sqrt{\mathrm{MSE}}$ than UVoW does. 


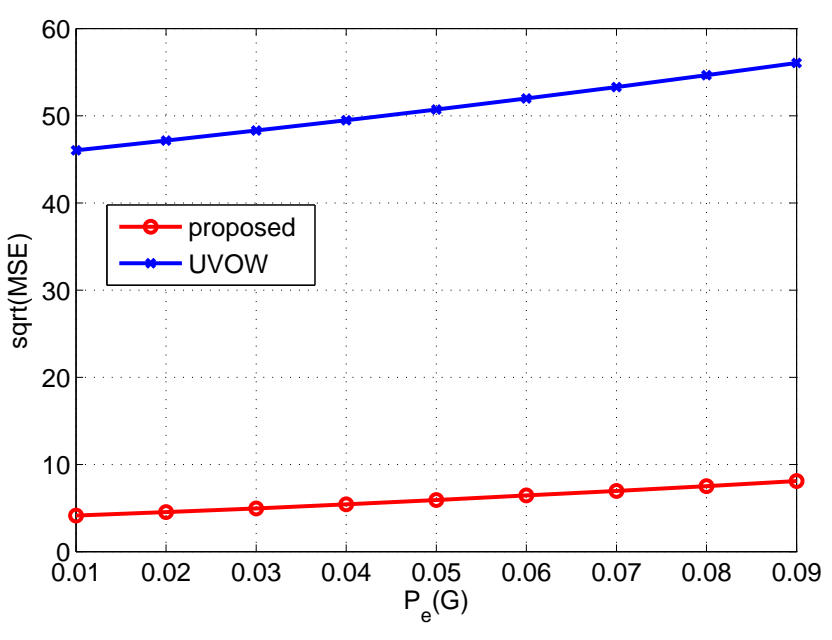

Figure 7: $\sqrt{\mathrm{MSE}}$ vs. packet loss rate at the good state $P_{e}(G)$.

Fig. 7 demonstrates the relationship between the expected $\sqrt{\mathrm{MSE}}$ and $P_{e}(G)$. As $P_{e}(G)$ is increased, the probability of a packet being lost in the good state is increased, which means the error correcting capability of the bits of the highest priorities should be enhanced by re-segmentation of the bits. As Fig. 7 shows, the characteristic of adaptivity enables the proposed scheme outperform UVoW in terms of $\sqrt{\mathrm{MSE}}$ for the entire range of $P_{e}(G)$ examined in the study. The average $\sqrt{\mathrm{MSE}}$ of the proposed scheme is 44.8486 lower than that of UVoW. This result also indicates that our proposed scheme is extremely suitable for the case that $P_{e}(G)$ is comparatively high during communication.

In the next simulation, an example video image is used in our performance test. The optimal solutions of $\left\{S, m_{1}\right.$, $\left.\cdots, m_{S}, H\right\}$ corresponding to the lowest expected $\sqrt{\mathrm{MSE}}$ under given channel parameters are tested in our scheme. The entire image is divided into many small images and we show only $1 / 16$ of the small images. Thus we obtain a partial image as shown in Fig. 8, whose size is $1 / 16$ of that of the entire image. Note that the size of the entire image will be too large to fit into the paper, and the quality of these small images is representative since all the small images are treated equally in uncompressed videos. The partial image in Fig. 8(a) is the original one; the partial images in Figs. 8(b)(d) are produced by the proposed scheme and the partial images in Figs. 8(c)(e) are produced by UVoW.

As shown in these figures, when the channel condition is comparatively good, both the proposed scheme and UVoW yield good quality, since all the data streams can be decoded. However, as the channel condition turns bad, the degradation of image quality is much more serious in UVoW. When the channel is in a bad condition (i.e., $P_{b \mid g}=0.1$ ), the sub-images produced by our scheme are still recognizable and hold a comparatively high quality, while a portion of the sub-images are black using UVoW. Under such bad channels, the most important bits can still be received and decoded in our scheme, but are completely lost in UVoW. This is because the protection to the most important bits is adaptively enhanced after re-segmentation to adapt to the degraded channel condition. These results clearly demonstrate the advantages of being adaptive both at the MD video coder and at the transmission scheduler to the varying channel conditions.

\section{CONCLUSION}

In this paper, we investigated the problem of streaming uncompressed HD video over $60 \mathrm{GHz}$ wireless networks. We developed an MD-FEC coding scheme that partitions the pixel bits and an interleaving based transmission strategy to optimize the expected $\sqrt{\mathrm{MSE}}$ of the reconstructed video quality. The performance of the proposed scheme was evaluated with simulations and shown to outperform an existing scheme with considerable gains.

\section{Acknowledgments}

This work is supported in part by the US National Science Foundation under Grants CNS-0953513 and CNS-1320664, any opinions, findings, and conclusions or recommendations expressed in this material are those of the author(s) and do not necessarily reflect the views of the foundation.

\section{REFERENCES}

[1] A. Albanese, J. Blömer, J. Edmonds, M. Luby, and M. Sudan. Priority encoding transmission. IEEE IEEE Trans. Inform. Theory, 42(6):1737-1744, Nov. 1996.

[2] B. Bosco. Adaptive cross layer design and implementation for Gigabit multimedia applications using $60 \mathrm{GHz}$ wireless links. Master's thesis, Arizona State Univ., Phoenix, AZ, 2011.

[3] M. X. Gong, D. Akhmetov, R. Want, and S. Mao. Directional CSMA/CA protocol with spatial reuse for mmWave wireless networks. In Proc. IEEE GLOBECOM 2010, pages 1-5, Miami, FL, Dec. 2010.

[4] W. Lee, K. Noh, S. Kim, and J. Heo. Efficient cooperative transmission for wireless $3 \mathrm{D}$ HD video transmission in $60 \mathrm{GHz}$ channel. IEEE Trans. Consum. Electron., 56(4):2481-2488, Nov. 2010.

[5] M. Manohara, R. Mudumbai, J. Gibson, and U. Madhow. Error correction scheme for uncompressed HD video over wireless. In Proc. IEEE ICME 2009, pages 802-805, New York, NY, June/July 2009.

[6] S. Mao, D. Bushmitch, S. Narayanan, and S. S. Panwar. MRTP: A multi-flow real-time transport protocol for ad hoc networks. IEEE Trans. Multimedia, 8(2):356-369, Apr. 2006.

[7] S. Mao, X. Cheng, Y. T. Hou, and H. D. Sherali. Multiple description video multicast in wireless ad hoc networks. ACM/Springer Mobile Netw. Appl., 11(1):63-73, Jan. 2006.

[8] S. Mao, S. Lin, S. Panwar, Y. Wang, and E. Celebi. Video transport over ad hoc networks: Multistream coding with multipath transport. IEEE J. Sel. Areas Commun., 21(10):1721-1737, Dec. 2003.

[9] S. Mao, S. Lin, Y. Wang, S. S. Panwar, and Y. Li. Multipath video transport over wireless ad hoc networks. IEEE Wireless Commun., 12(4):42-49, Aug. 2005.

[10] T. S. Rappaport, J. N. Murdock, and F. Gutierrez. State of the art in $60-\mathrm{GHz}$ integrated circuits and systems for wireless communications. Proc. IEEE, 99(8):1390-1436, Aug. 2011. 


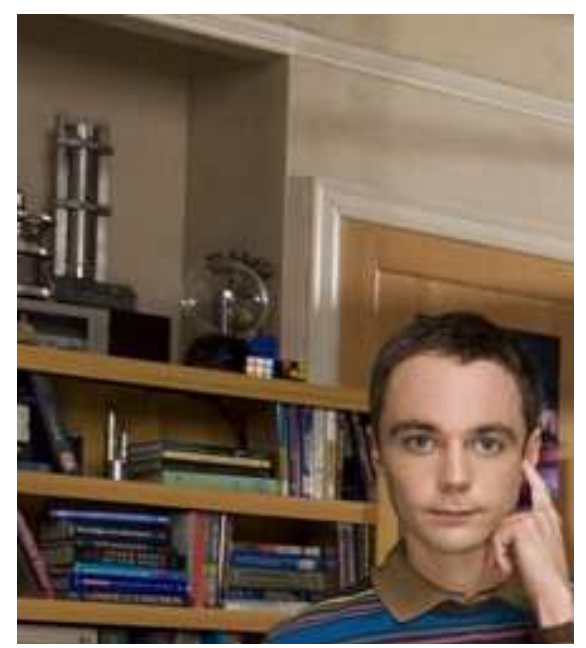

(a)

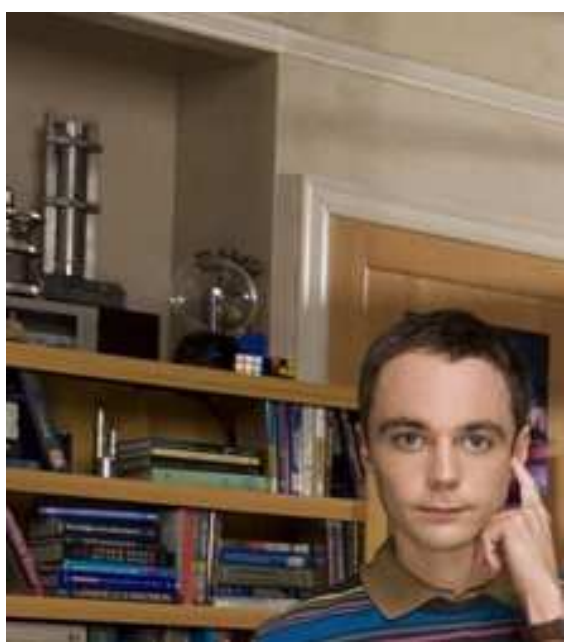

(b)

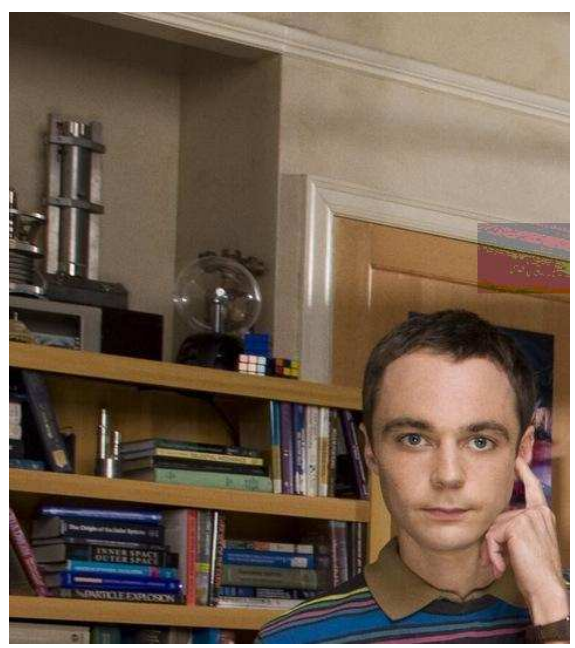

(d)

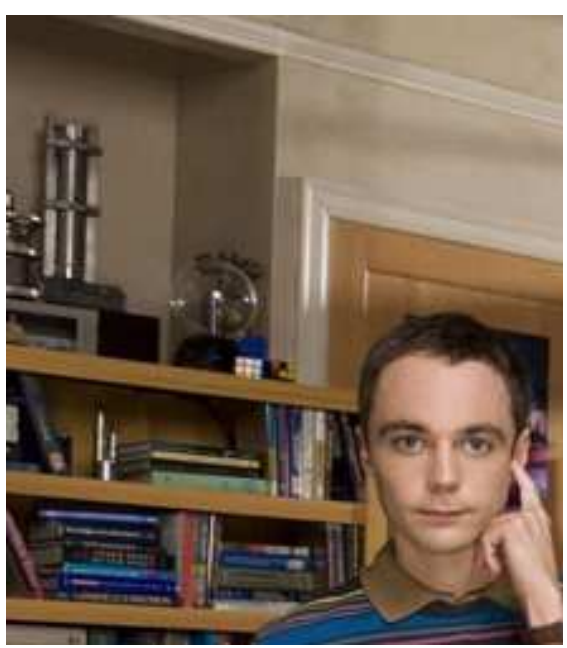

(c)

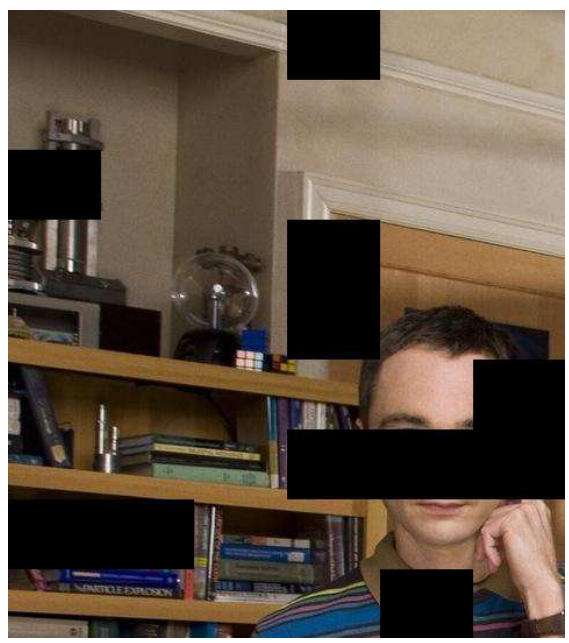

(e)

Figure 8: Visual quality comparison of the two schemes under different channel conditions. (a): the original image. (b)(d): the proposed scheme. (c)(e): UVoW. (b)(c) $P_{b \mid g}=0.3 . \quad$ (d)(e) $P_{b \mid g}=0.1 . \quad P_{g \mid b}=0.1$ and $P_{e}(G)=0.01$ are fixed in all the simulations.

[11] L. Rizzo. Effective erasure codes for reliable computer communication protocols. ACM Computer Commun. Rev., 27(2):24-36, Apr. 1997.

[12] H.-R. Shao and et al. Adaptive multi-beam transmission of uncompressed video over $60 \mathrm{GHz}$ wireless systems. In Proc. Future Generation Commun. Netw. 2007, pages 430-435, Jeju-Island, South Korea, Dec. 2007.

[13] H. Singh, X. Qin, H. Shao, C. Ngo, C. Kwon, and S. S. Kim. Support of uncompressed video streaming over $60 \mathrm{GHz}$ wireless networks. In Proc. IEEE CCNC 2008, pages 243-248, Las Vegas, NV, Jan. 2008.

[14] I.-K. Son, S. Mao, M. Gong, and Y. Li. On frame-based scheduling for directional mmWave WPANs. In Proc. IEEE INFOCOM 2012, pages 2149-2157, Orlando, FL, Mar. 2012.

[15] Y. Wang, A. Reibman, and S. Lin. Multiple description coding for video delivery. Proc. IEEE, 93(1):57-70, Jan. 2005.
[16] L. R. Wilhelmsson and L. B. Milstein. On the effect of imperfect interleaving for the Gilbert Elliott channel. IEEE Trans. Commun., 47(5):681-688, May 1999.

[17] Y. Zhao, S. Mao, J. Neel, and J. H. Reed. Performance evaluation of cognitive radios: metrics, utility functions, and methodologies. Proc. IEEE, 97(4):642-659, Apr. 2009. 